\title{
BMJ Open Variation in use of non-surgical treatments among osteoarthritis patients in orthopaedic practice in the Netherlands
}

\author{
Stefanie N Hofstede, ${ }^{1}$ Thea P M Vliet Vlieland, ${ }^{2}$ Cornelia H M van den Ende, ${ }^{3}$
} Rob G H H Nelissen, ${ }^{2}$ Perla J Marang-van de Mheen, ${ }^{1}$ Leti van Bodegom-Vos ${ }^{1}$

To cite: Hofstede SN, Vliet Vlieland TPM, van den Ende CHM, et al. Variation in use of non-surgical treatments among osteoarthritis patients in orthopaedic practice in the Netherlands. BMJ Open 2015:5:e009117. doi:10.1136/ bmjopen-2015-009117

- Prepublication history for this paper is available online. To view these files please visit the journal online (http://dx.doi.org/10.1136/ bmjopen-2015-009117).

Received 18 June 2015 Revised 12 August 2015 Accepted 25 August 2015

CrossMark

${ }^{1}$ Department of Medical Decision Making, Leiden University Medical Center Leiden, The Netherlands ${ }^{2}$ Department of Orthopaedics, Leiden University Medical Center, Leiden,

The Netherlands

${ }^{3}$ Department of

Rheumatology, Sint

Maartenskliniek Nijmegen,

Ubbergen, The Netherlands

Correspondence to Dr Leti van Bodegom-Vos; I.vanbodegom-vos@lumc.nl

\section{ABSTRACT}

Objectives: National and international evidence-based guidelines for hip and knee osteoarthritis $(\mathrm{OA})$ recommend to start with non-surgical treatments, followed by surgical intervention if a patient does not respond sufficiently to non-surgical treatments, but there are indications that these are not optimally used. The aim of this study was to assess the extent to which all recommended non-surgical treatments were used by patients with hip or knee $\mathrm{OA}$ who receive(d) a total hip or knee replacement, as reported by patients and orthopaedic surgeons.

Setting: We performed two cross-sectional internetbased surveys among patients and orthopaedic surgeons throughout the Netherlands.

Participants: 195 OA patients either have undergone total knee arthroplasty or total hip arthroplasty no longer than 12 months ago or being on the waiting list for surgery with a confirmed date within 3 months and 482 orthopaedic surgeons were invited to participate.

Primary and secondary outcome measures: The use of recommended non-surgical treatments including education about OA/treatment options, lifestyle advice, dietary therapy, physical therapy, acetaminophen, NSAIDs and glucocorticoid injections.

Results: 174 OA patients (93\%) and 172 orthopaedic surgeons $(36 \%)$ completed the surveys. Most recommended non-surgical treatments were given to the majority of patients (eg, $80 \%$ education about $\mathrm{OA}$, $73 \%$ physical therapy, $72 \%$ acetaminophen, $80 \%$ NSAIDs). However, only $6 \%$ of patients and $10 \%$ of orthopaedic surgeons reported using a combination of all recommended treatments. Dietary therapy was used least frequently. Only $11 \%$ of overweight and $30 \%$ of obese participants reported having received dietary therapy and $28 \%$ of orthopaedic surgeons reported to prescribe dietary therapy to overweight patients.

Conclusions: While most recommended non-surgical treatments were used frequently as single therapy, the combination is used in only a small percentage of OA patients. Especially, use of dietary therapy may be improved to help patients manage their symptoms, and potentially delay the need for joint arthroplasty.

\section{Strengths and limitations of this study}

This study assesses the use of non-surgical treatments in orthopaedic practice, which has not been done before.

- This study includes the perspective of orthopaedic surgeons and patients.

- Owing to the retrospective nature of our study and the reliance on self-reported data, it is susceptible to recall bias.

\section{INTRODUCTION}

Patients with symptomatic hip or knee osteoarthritis (OA) suffer from pain and loss of function for which treatment is required. Different treatment options are available, surgical and non-surgical treatments. For patients, good quality of care is achieved if their symptoms are managed in the short term, but also taking into account which treatment option results in the best longterm patient outcomes. As the lifespan of a prosthesis is limited, and patient outcomes after revision arthroplasty are not as good as after primary surgery, ${ }^{1}$ it is generally acknowledged that total hip arthroplasty (THA) and total knee arthroplasty (TKA) should not be performed too early. For this reason, evidence-based guidelines recommend to start with non-surgical treatments (eg, education, physical therapy, non-steroidal antiinflammatory drugs (NSAIDs)), ${ }^{2-6}$ and to use surgical intervention only if a patient does not respond sufficiently to non-surgical treatment options in the context of end stage $\mathrm{OA}^{7-13}$ These recommendations are all based on the large body of evidence supporting the effectiveness of these non-surgical treatments to help patients with hip and knee OA manage their symptoms and preserve joint function. ${ }^{14}$

Despite these guidelines, several studies have suggested that the use of non-surgical 
treatments in patients with hip or knee OA can be improved. $^{7}{ }^{15-18}$ For instance, Snijders et $a l^{7}$ demonstrated that $81 \%$ of patients with hip or knee OA did not receive all non-surgical treatments in the primary care setting. However, patients with OA may receive nonsurgical treatments later on in the care trajectory from their orthopaedic surgeon once referred to specialist care. In the Netherlands, patients with OA are usually treated by the general practitioner. According to guidelines, patients should be referred to the orthopaedic surgeon if they do not respond sufficiently to nonsurgical treatment options. In orthopaedic practice, the decision will be made to start/continue non-surgical treatments or to perform a surgery depending on previous received treatments and disease severity. Therefore, orthopaedic surgeons have an important role in ensuring optimal care of patients with OA by confirming whether recommended non-surgical treatments have been exhaustedly used before considering a surgical intervention. Furthermore, surgical interventions, like a THA and TKA do not have good patient reported outcome in about $10-20 \%$ of patients. ${ }^{19-22}$ This stresses even more the importance of good non-surgical treatment modalities before implant surgery is encountered. McHugh $e t a l^{23}$ examined which treatments patients with OA used throughout the care trajectory, before and while on the waiting list for a TKA or THA, and showed that only $10 \%$ of the patients had received information on pain management with the consequence that some patients used their own regime to take analgesics. This in turn may have led to insufficient effects of analgesics. However, they did not investigate the full range of nonsurgical treatments and the estimates were reported by patients, so that the information may have been given to them but not remembered. Given the known effectiveness of non-surgical treatments individually and for some combinations (eg, physical therapy with dietary therapy $^{24}$ ), use of the full range of available recommended non-surgical treatments may improve patient outcomes, ${ }^{2-6}$ thereby improving quality of care, and postpone the need for surgery which would reduce chances for a revision with worse patient outcomes than primary surgery. What is currently lacking and needed to provide a complete view, is an assessment of the full range of non-surgical treatments as well as including both patients' and orthopaedic surgeons' perspectives, as these may differ.

Therefore, the aim of this study was to assess the extent to which all recommended non-surgical treatments were used by patients with hip or knee $\mathrm{OA}$ as reported by patients and orthopaedic surgeons, both as a single option and in combination.

\section{MATERIALS AND METHODS}

\section{Study design}

We performed two cross-sectional internet-based surveys in November and December 2013 and January 2014 to assess the use of non-surgical treatments in orthopaedic practice as reported by patients and orthopaedic surgeons.

\section{Population}

\section{Patients}

A total of 195 patients were invited by email to participate in the survey, to estimate a previously reported $19 \%$ use of non-surgical treatments among 47000 patients with hip and knee OA annually in the Netherlands, ${ }^{25}$ with a 5\% margin of error. Patients were recruited via advertisements in local newspapers across the Netherlands, and through the websites or newsletters of patient associations. Patients who volunteered to participate in the survey in reaction to the advertisements were dialed by the research team to provide information about the study, to answer questions and to ask whether they approved for participation. In addition, patients received written information before the start of the survey and the availability to stop during the study. In addition, patients received written information before the start of the survey and the availability to stop during the study. Inclusion criteria for patients were: age $\geq 18$ years, a doctor's diagnosis of hip or knee OA, and either have undergone TKA or THA no longer than 12 months ago or being on the waiting list for surgery with a confirmed date within 3 months. Patients who were unable to understand written Dutch or who had undergone or were scheduled for revision surgery were excluded from the study. Patients who initially indicated that they wanted to participate but did not respond, were sent two reminders, one after one and a half weeks, and if still no response again after 3 weeks. Participants who completed the questionnaire received a $€ 10$ gift card as an incentive.

\section{Orthopaedic surgeons}

All 482 Dutch orthopaedic surgeons listed in the registry of the Netherlands Orthopaedic Association (NOV) and/or the Dutch medical address book with an email address received an invitation to participate. All orthopaedic surgeons who treated patients with hip or knee OA were eligible. Orthopaedic surgeons who did not respond received two reminders, one after one and a half weeks and if still no response again after 3 weeks. Orthopaedic surgeons did not receive an incentive for their participation.

\section{Survey development \\ Survey for patients}

The survey for patients included questions about general patient characteristics, general health and symptoms of OA, and non-surgical treatment for OA. Patient characteristics included: age, gender, region of residence (north, middle, and south), educational level (basic education (none or only primary education), intermediate education (prevocational secondary education, senior secondary vocational training, senior secondary general education, pre-university education), or high education 
(higher professional education or university (bachelor, master, or PhD degree)), work situation (paid work or not), height $(\mathrm{cm})$ and weight $(\mathrm{kg})$ to calculate the body mass index (BMI), and type of insurance (basic and/or additional coverage for care such as physical therapy, glucosamine sulfate and hyaluronic acid). Furthermore, the survey included general and disease-specific health questions, such as duration of OA and duration of complaints of the affected joint, comorbidities, average pain during 6 months before surgery, measured on a 0 (no pain)-10 (unbearable pain) scale, and patient-perceived reasons for surgery.

Questions about healthcare use included all nonsurgical options before joint replacement surgery as described in the Dutch stepped-care strategy (SCS) and were formulated as follows: "Did you receive the following treatments for the complaints of your affected joint before joint replacement surgery?." The SCS is based on (inter)national guidelines. ${ }^{26}{ }^{27}$ The first step consists of education, life style advice and acetaminophen. If the treatment options in the first step are not sufficient, treatment options in the second step can be considered (exercise therapy, dietary therapy and NSAIDs).

Multidisciplinary care, intra-articular injections, and transcutaneous electrical nerve stimulation (TENS) are treatment options in the third step and could be considered if treatment options in step one or two are ineffective.

In the survey these non-surgical treatments were formulated as follows: education about the disease OA, education about the possible treatment options in OA, lifestyle advices (ie, stay active, lose weight), physical therapy/ exercise therapy, acetaminophen, anti-inflammatory painkillers (eg, NSAIDS such as Celebrex, Diclofenac, Cataflam, Voltaren), tramadol (eg, Tramal, Tramagetic, Tradonal, Zaldiar), multidisciplinary care (care of different healthcare providers at the same time, eg, in a revalidation centre), injections in the knee, TENS (therapy that uses electrical current on the skin). Patients could choose one or more of the following answers: yes, received from the orthopaedic surgeon; yes, received from another healthcare provider; yes, received on my own initiative; no.

\section{Survey for orthopaedic surgeons}

The survey for orthopaedic surgeons included questions about their background characteristics, and the prescription of non-surgical treatments. Characteristics of orthopaedic surgeons included: age, gender, work region, work setting, years of working experience as an orthopaedic surgeon, number of new patients with hip/knee OA seen per month. Questions about prescribed treatments included all non-surgical options described in the SCS $^{26} 27$ and were formulated as follows: "If patients did not receive the following non-surgical treatments, do you prescribe these treatments?" In case of physical therapy or dietary therapy we asked whether they referred patients, rather than initiating this treatment themselves. Orthopaedic surgeons could choose one of the following answers: never, sometimes, often or (almost) always.

\section{Analysis}

Descriptive statistics were used to describe the characteristics of respondents, and the use of non-surgical treatments from the patients' or orthopaedic surgeons' perspectives. From the patient perspective we distinguished the use of non-surgical treatments prescribed by any healthcare provider, by the orthopaedic surgeon, or undertaken by their own initiative. From the orthopaedic surgeon perspective we dichotomised the answers into 'prescribed' (often/almost always) and 'not prescribed' (never/sometimes).

To assess the use of non-surgical treatments, we made a distinction between non-surgical treatments recommended by various organisations (eg, OARSI, EULAR, AAOS, NOV) ${ }^{14} 16$ and other non-surgical treatments. The recommended non-surgical treatments were education about OA, education about different treatment options, lifestyle advice, (referral to) dietary therapy, physical therapy containing exercises, acetaminophen, NSAIDs and glucocorticoid injections. Other nonsurgical treatments included glucosamine sulfate, tramadol, multidisciplinary care, TENS, and hyaluronic acid injections (for knee OA). These treatments are not supported by high quality evidence or clinical guidelines, but are nevertheless sometimes recommended and used by patients with OA. BMI of patients was classified into normal weight if BMI $<25 \mathrm{~kg} / \mathrm{m}^{2}$, overweight if BMI $\geq 25$ $<30 \mathrm{~kg} / \mathrm{m}^{2}$, and obese if BMI $\geq 30 \mathrm{~kg} / \mathrm{m}^{2}$ to assess whether dietary therapy was indicated. If BMI was unknown, we assumed that dietary therapy was not indicated for that patient.

For each non-surgical treatment, we calculated the percentage of patients who had received this treatment, and the percentage of orthopaedic surgeons who always/ often prescribed this treatment for their patients. In addition, we calculated the percentage of participants who received/prescribed the recommended non-surgical treatments listed in each step of the Dutch SCS including the previous steps (conditional percentage). The proportion of patients and orthopaedic surgeons using each non-surgical treatment was compared using the $\chi^{2}$ test.

In addition, we explored whether patients and orthopaedic surgeons using all recommended treatments differed from those who did not, in age, gender, region of residence, BMI and level of education (for patients) and on differences in age, gender, work region, work setting, years of working experience and number of new patients with hip/knee OA seen per month (for orthopaedic surgeons). We also explored differences in use of each treatment between patients with THA and TKA. The independent $\mathrm{t}$ test or Mann Whitney $\mathrm{U}$ tests for continuous variables and $\chi^{2}$ tests or Fisher's exact tests for proportions was used to compare differences between subgroups. Significance testing was done two-sided at $\alpha=0.05$. SPSS V.20.0 was used for analyses. 
Ethics

This study protocol was presented to the Medical Ethical Committee of the Leiden University Medical Center (CME P13.087/NV/nv). An exemption was obtained, as ethical approval for this type of study is not required under the Dutch law.

\section{RESULTS}

Response

A total of 182 patients (response rate of 93\%) completed the survey. Eight patients were subsequently excluded from the analyses, because they did not fulfil the inclusion criteria. This left 174 patients (89\%) included in the final analyses.

One hundred and eighty one (response rate of 38\%) orthopaedic surgeons completed the questionnaire. Nine orthopaedic surgeons were excluded because they indicated they did not see patients with OA in consultations. Thus a total of $172(36 \%)$ orthopaedic surgeons were included in the final analyses.

\section{Characteristics of the population}

Patients

Characteristics of patients who completed the questionnaire are described in table 1 . The majority of the participants were female, $26 \%$ were obese (BMI $\geq 30 \mathrm{~kg} / \mathrm{m}^{2}$ ) and thus indicated for dietary therapy. Most of the respondents had already undergone THA or TKA at the time of recruitment, and a significant proportion of patients reported a duration of symptoms for more than 5 years. Almost all patients had additional insurance coverage, meaning that physical and dietary therapy was likely to be covered by their insurance rather than being subject to out of pocket expenses.

\section{Orthopaedic surgeons}

The characteristics of the orthopaedic surgeons who completed the questionnaires are presented in table 2. On average, they had been working for 13 years (SD 8) as an orthopaedic surgeon, and saw an average of 25 new patients with hip OA (SD 24) and 31 (SD 22) new patients with knee OA per month. Orthopaedic surgeons from various parts of the country and different hospital types were included in the sample.

\section{Use of recommended non-surgical treatments}

Table 3 shows the percentage of patients that received recommended and non-recommended non-surgical treatments as reported by patients.

The most frequently received non-surgical treatments were education about OA $(80 \%)$, physical therapy $(73 \%)$, acetaminophen $(72 \%)$, education about different treatment options (66\%) and NSAIDs (64\%). Of these, education about $\mathrm{OA}$ and education about different treatment options were mostly received from the orthopaedic surgeon (table 3), whereas the other treatments were received from another healthcare
Table 1 Characteristics of included patients with hip or knee OA

\begin{tabular}{|c|c|}
\hline Characteristics & $\begin{array}{l}\text { Patients } \\
(n=174)\end{array}$ \\
\hline Age in years (mean, SD) & $64(7.7)$ \\
\hline Female, n (\%) & $125(72)$ \\
\hline \multicolumn{2}{|l|}{$\mathrm{BMI}, \mathrm{n}(\%)$} \\
\hline$<25$ & $36(21)$ \\
\hline$\geq 25<30$ & $84(48)$ \\
\hline$\geq 30$ & $46(26)$ \\
\hline Missing & $8(5)$ \\
\hline Knee OA, n (\%) & $94(54)$ \\
\hline \multicolumn{2}{|c|}{ Joint replacement (THA or TKA), n (\%) } \\
\hline Yes & $169(97)$ \\
\hline Within 3 months & $5(3)$ \\
\hline First joint replacement, n (\%) & $132(73)$ \\
\hline \multicolumn{2}{|c|}{ Duration of complaints of affected hip/ knee, $n(\%)$} \\
\hline$<1$ year & $13(8)$ \\
\hline $1-5$ years & $86(49)$ \\
\hline $6-10$ years & $42(24)$ \\
\hline $11-20$ years & $25(14)$ \\
\hline$>20$ years & $8(5)$ \\
\hline Pain before surgery, mean $(\mathrm{SD})^{\star}$ & $7.16(1.8)$ \\
\hline \multicolumn{2}{|l|}{ Comorbidities, n (\%) } \\
\hline Diabetes & $10(6)$ \\
\hline $\begin{array}{l}\text { Stroke, cerebral haemorrhage, } \\
\text { cerebral infarct }\end{array}$ & $5(3)$ \\
\hline Cancer & $10(6)$ \\
\hline Cardiovascular disease & $12(7)$ \\
\hline Migraine or severe headaches & $17(10)$ \\
\hline High blood pressure & $57(33)$ \\
\hline $\begin{array}{l}\text { Asthma, chronic bronchitis, } \\
\text { emphysema }\end{array}$ & $14(8)$ \\
\hline Chronic joint inflammation & $32(18)$ \\
\hline Other & $20(11)$ \\
\hline \multicolumn{2}{|l|}{ Education, n (\%) } \\
\hline Basic & $6(3)$ \\
\hline Intermediate & $120(69)$ \\
\hline High & 47 (27) \\
\hline Missing & $1(1)$ \\
\hline Paid work, yes n (\%) & 55 (32) \\
\hline \multicolumn{2}{|l|}{ Region of residence, n (\%) } \\
\hline North & $70(40)$ \\
\hline Middle & $55(32)$ \\
\hline South & $49(28)$ \\
\hline \multicolumn{2}{|l|}{ Living situation, n (\%) } \\
\hline Live alone & $39(22)$ \\
\hline Live with others & $135(78)$ \\
\hline \multicolumn{2}{|l|}{ Type of insurance, n (\%) } \\
\hline Basic only & $4(2)$ \\
\hline Basic with additional coverage & $170(98)$ \\
\hline \multicolumn{2}{|c|}{$\begin{array}{l}\text { “Average pain during } 6 \text { months before surgery measured on a } \\
\text { (no pain)-10 (unbearable pain) scale. } \\
\text { BMI, body mass index; OA, osteoarthritis; THA, total hip } \\
\text { arthroplasty; TKA, total knee arthroplasty. }\end{array}$} \\
\hline
\end{tabular}

professional, or patients own initiative. Dietary therapy was used least frequently, even when non-overweight patients were excluded. Only $11 \%$ of overweight patients and $30 \%$ of the obese patients reported they had received dietary therapy. A minority of these patients was 
Table 2 Characteristics of orthopaedic surgeons who treated patients with hip or knee OA

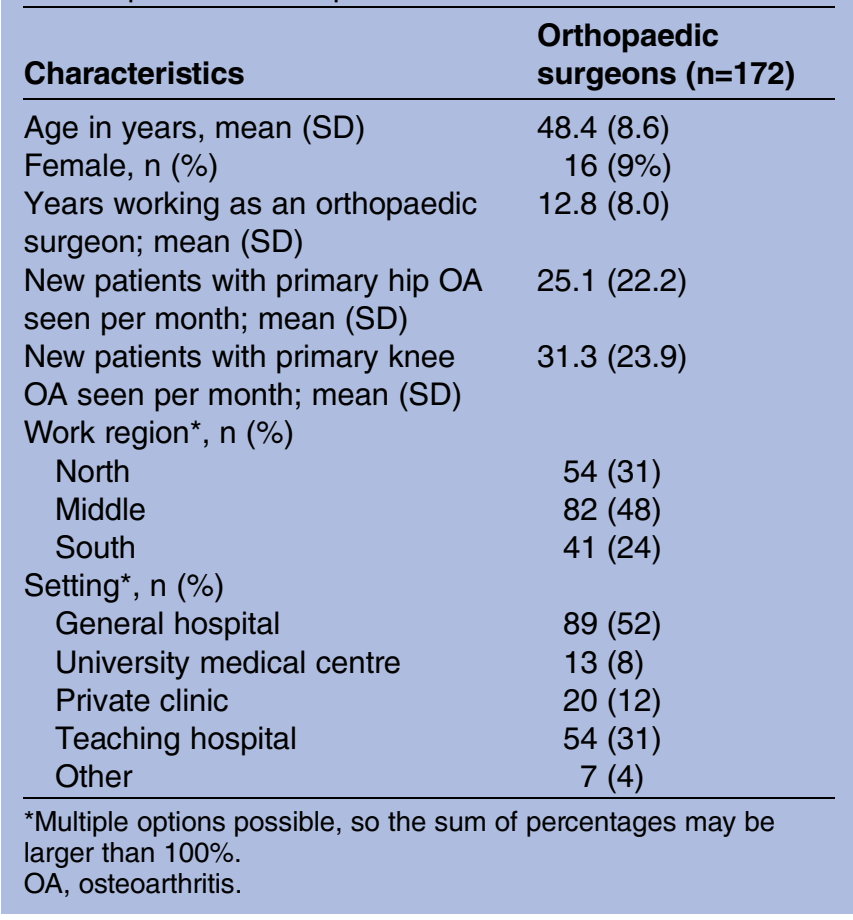

referred to a dietician by their orthopaedic surgeon (table 3).

In addition, looking at the conditional percentage in table 4, only $33 \%$ of the patients received all recommended treatments in step 1 of the SCS, and eleven
(6\%) patients reported to have received all recommended treatments in step 1 and 2 of the SCS. Because many patients did not remember which type of injection they received, we excluded glucocorticoid injections and did not calculated the conditional percentage of step 1 , 2 and 3 together.

Reasons for surgery according to patients (multiple answers possible) were: pain could not be controlled with painkillers (55\% of the patients), insufficient effect of other treatments (eg, physical therapy, dietary advice) $(51 \%)$, duration of symptoms $(41 \%)$, difficulties with daily activities (75\%), or other reasons (17\%) (eg, severe cartilage loss, difficulties with sports, immobility).

Table 5 shows the percentage of orthopaedic surgeons that often or always prescribes recommended nonsurgical treatments as reported by orthopaedic surgeons. Orthopaedic surgeons often reported to prescribe lifestyle advice $(98 \%)$, education about different treatment options $(95 \%)$, education about OA $(87 \%)$ and acetaminophen $(64 \%)$. However, table 6 shows that only 96 $(56 \%)$ of the orthopaedic surgeons reported prescribing all recommended treatments in step 1 of the SCS, 17 $(10 \%)$ reported prescribing all recommended treatments in step 1 and 2, and $10(6 \%)$ reported prescribing all recommended treatments in step 1, 2 and 3, if the patient had not received these treatments in their previous care trajectory. As among patients, dietary therapy was reported as the least prescribed treatment (reported by $28 \%$ of the orthopaedic surgeons), followed by intra-articular injections $(43 \%)$ and physical therapy $(54 \%)$.

Table 3 Received recommended and non-recommended non-surgical treatments by patients as reported by patients

\begin{tabular}{|c|c|c|c|c|}
\hline \multirow[b]{2}{*}{ Non-surgical treatment } & \multirow[b]{2}{*}{$\begin{array}{l}\text { Received, } \\
\text { n (\%) }\end{array}$} & \multicolumn{3}{|l|}{ Received by ${ }^{\star}$} \\
\hline & & $\begin{array}{l}\text { Orthopaedic } \\
\text { surgeon, n (\%) }\end{array}$ & $\begin{array}{l}\text { Other healthcare } \\
\text { professional, n (\%) }\end{array}$ & $\begin{array}{l}\text { Own initiative, } \\
\mathrm{n}(\%)\end{array}$ \\
\hline Education about OA & $139(80)$ & $95(68)$ & $30(22)$ & $31(22)$ \\
\hline $\begin{array}{l}\text { Education about different } \\
\text { treatment options }\end{array}$ & $115(66)$ & $80(70)$ & $27(23)$ & $21(18)$ \\
\hline Lifestyle advice & $107(62)$ & $37(35)$ & $40(37)$ & $40(37)$ \\
\hline \multicolumn{5}{|l|}{ Dietary therapy, if indicated } \\
\hline $\mathrm{BMI} \geq 25<30$ & $9(11)$ & $2(22)$ & $3(33)$ & $5(56)$ \\
\hline $\mathrm{BMI} \geq 30$ & $14(30)$ & $1(7)$ & $7(50)$ & $6(43)$ \\
\hline Physical therapy & $127(73)$ & $33(26)$ & $70(55)$ & $36(28)$ \\
\hline Contained exercises & $116(91)$ & & & \\
\hline Acetaminophen & $125(72)$ & $26(21)$ & $30(24)$ & $73(58)$ \\
\hline NSAIDs & $111(64)$ & $43(39)$ & $51(46)$ & $19(17)$ \\
\hline Tramadol & $44(25)$ & $21(48)$ & $24(55)$ & $1(2)$ \\
\hline Glucosamine sulfate & $58(33)$ & 7 (12) & 9 (16) & $46(79)$ \\
\hline Multidisciplinary care & $12(7)$ & 4 (33) & $8(67)$ & $1(8)$ \\
\hline TENS & $10(6)$ & 0 & $10(100)$ & 0 \\
\hline $\begin{array}{l}\text { Intra-articular injections } \\
\text { (knee } O A n=94)\end{array}$ & $54(57)$ & $46(85)$ & $12(22)$ & 0 \\
\hline Glucocorticoid & $28(30)$ & & & \\
\hline Hyaluronic acid & $7(7)$ & & & \\
\hline Unknown & $20(37)$ & & & \\
\hline
\end{tabular}

*Multiple options possible, thus the sum of orthopaedic surgeon, other healthcare professional and own initiative can be $>100 \%$.

BMI, body mass index; NSAIDs, non-steroidal anti-inflammatory drugs; OA, osteoarthritis; TENS, transcutaneous electrical nerve stimulation. 
Table 4 Conditional percentage of patients receiving recommended non-surgical treatments in stepped care strategy as reported by patients

Recommended non-surgical treatments in stepped care strategy

Conditional $\mathbf{n}(\%)$

Step 1: education about OA+education about different treatment options+lifestyle advice+acetaminophen 57 (33)

Step 1+2: step 1+(Referral to) dietary therapy, when indicated+physical therapy+NSAIDs

Step 1, 2+3: step 1+2+intra-articular injections (for knee OA $n=94$ )

$\mathrm{n} / \mathrm{c}$

$\mathrm{n} / \mathrm{c}$ : not calculated, because many patients did not know which type of injection they received.

NSAIDs, non-steroidal anti-inflammatory drugs; OA, osteoarthritis.

No differences were found between patients receiving and not receiving all recommended non-surgical treatments in any of the patient characteristics tested. Similarly, no differences were found between orthopaedic surgeons prescribing and not prescribing all recommended non-surgical treatments in any of their characteristics. Patients with knee OA received acetaminophen more often than patients with hip OA $(80 \%$ vs $63 \%$ respectively $(\mathrm{p}=0.01)$ ), but no differences were found in the use of other treatments. Furthermore, comparing patients and orthopaedic surgeons, no differences were found in the proportion using education about OA $(p=0.09)$, acetaminophen $(p=0.18)$, NSAIDs $(p=0.39)$, and the percentage using all recommended non-surgical treatments $(p=0.22)$. A smaller percentage of patients compared to orthopaedic surgeons reported having received/prescribed education about different treatment options $(p<0.001)$, lifestyle advice $(p<0.001)$ and dietary therapy $(\mathrm{p}=0.03)$. The use of physical therapy on the other hand was reported to have been received by more patients than being prescribed by orthopaedic surgeons $(73 \%$ vs $54 \%(\mathrm{p}<0.001))$.

\section{Use of other non-surgical treatments}

Glucosamine sulfate was the most frequently used other non-surgical treatment, reported by $33 \%$ of patients, and mostly (79\%) used on their own initiative. Multidisciplinary care $(7 \%)$ and TENS $(6 \%)$ were the least often reported other treatments. Thirty-three per

Table 5 Prescribed recommended non-surgical treatments, as reported by orthopaedic surgeons (often or always)

\begin{tabular}{ll}
\hline $\begin{array}{l}\text { Recommended non-surgical } \\
\text { treatments }\end{array}$ & $\begin{array}{l}\text { Population } \\
\mathbf{n}(\%)\end{array}$ \\
Participants & 172 \\
\hline Education about OA & $149(87)$ \\
Education about different treatment options & $163(95)$ \\
Lifestyle advice & $168(98)$ \\
(Referral to) dietary therapy, when & $49(28)$ \\
indicated & \\
Physical therapy & $93(54)$ \\
Acetaminophen & $112(64)$ \\
NSAIDs & $102(59)$ \\
Intra-articular injections & $74(43)$ \\
\hline NSAIDs, non-steroidal anti-inflammatory drugs; OA, osteoarthritis.
\end{tabular}

cent of the patients who received multidisciplinary care were referred by their orthopaedic surgeon, and none of the patients who used TENS was referred by their orthopaedic surgeon. Overall, orthopaedic surgeons rarely prescribed any of these treatments not recommended by published OA guidelines, the highest percentage $(8 \%)$ was for the recommendation of glucosamine sulfate.

\section{DISCUSSION}

Our study showed that although most recommended non-surgical treatments seem to be frequently used as a single option in OA patients who receive(d) a THA or TKA, only a small percentage of patients received all recommended non-surgical treatments. For that matter, only $6 \%$ of patients and $10 \%$ of orthopaedic surgeons reported using all recommended non-surgical treatments in step 1 and 2 of the SCS. ${ }^{27}$ Given the known effectiveness of each of these treatments individually, use of the full range of available modalities may improve patient outcomes. $^{2-6}$

Dietary therapy was the least frequently used recommended non-surgical treatment for OA. Only $11 \%$ of overweight patients and $30 \%$ of obese patients reported having received dietary therapy, and only $28 \%$ of orthopaedic surgeons reported they would prescribe dietary therapy. Another study in the Netherlands showed that only $14 \%$ of overweight and obese patients with OA reported receiving dietary therapy. ${ }^{16}$ This is even lower than reported in our study, but these patients were recruited by general practitioners, and thus may have subsequently received dietary therapy later on in the care trajectory for example, after referral to an orthopaedic surgeon. In our study, patients had visited multiple healthcare providers, potentially increasing the likelihood of being offered dietary therapy when indicated. In other countries, dietary therapy seems to be more commonly used, for example, $59 \%$ of physicians prescribed 'weight reduction' in a study performed in France, ${ }^{13}$ and $31 \%$ of patients with $\mathrm{OA}$ in a study in Canada. ${ }^{17}$ Although the numbers are higher, the overall low rates across studies suggest that there is room for improvement. Similarly, a considerable number of patients were not prescribed physical therapy. The use of physical therapy as a non-surgical treatment could even be overestimated, because orthopaedic surgeons 
Table 6 Conditional percentage of orthopaedic surgeons who prescribe recommended non-surgical treatments in stepped care strategy

Recommended non-surgical treatments

Conditional

in stepped care strategy

Step 1: education about OA+education about different treatment options+lifestyle advice +acetaminophen

Step 1+2: step 1+(referral to) dietary therapy, when indicated+physical therapy+NSAIDs $96(56)$

Step 1, 2+3: step 1+2+intra-articular injections (for knee OA n=94)

sometimes prescribe physical therapy as preparation before surgery instead of a non-surgical treatment to delay surgery. Dietary therapy and physical therapy are the only two recommended non-surgical treatments that an orthopaedic surgeon cannot provide himself, but for which referral is needed. Improving the use of these two treatments in orthopaedic care, may result in better quality of patient care as the combination of weight loss plus exercise is shown to provide better overall improvements in function, pain and mobility among older overweight and obese adults with knee OA compared with either intervention alone. ${ }^{24}$

This study has some limitations. First, because of the retrospective nature of our study and the reliance on self-reported data, it is susceptible to recall bias. In an attempt to reduce this bias, we limited inclusion to patients who had a TKA or THA no longer than 12 months ago, or were scheduled for surgery within the next 3 months. Second, the use of an internet-based survey could induce selection bias. It is known that the majority of THA and TKA patients prefer pen-and-paper questionnaires, and that patients who prefer electronic questionnaires differ from patients who prefer pen-and-paper questionnaires. ${ }^{28}$ It is possible that more elderly persons do not have internet or an email address compared to younger persons, which could have led to a selection of younger persons. The average age of patients with OA is 68.2 years $^{29}$ and our population is slightly younger, on average 64 (SD 7.7) years. However, age was not associated with the use of all recommended non-surgical treatments. Third, the use of a sample of patients responding to an advertisement may have introduced sampling bias. However, as our responding patients were distributed across different regions in the Netherlands, and had an age and gender distribution comparable to OA patients, we think that any bias that may have occurred is likely to be small. Similarly, selection bias may have occurred as a result of the low response rate $(38 \%)$ among orthopaedic surgeons. However, such a response rate is comparable or higher than found in other online surveys among orthopaedic surgeons in the Netherlands. ${ }^{30} 31$ It is possible that orthopaedic surgeons who are not interested in nonsurgical treatment were less likely to complete the questionnaire or that orthopaedic surgeons overestimate their use of non-surgical treatments. This would only lead to an overestimation of non-surgical treatment use and the use may be even lower. Furthermore, the use of acetaminophen, NSAIDs and tramadol could have been overestimated, as we were not able to define a minimum for the use of these treatments (eg, at least 1 tablet per day) due to differences between recommendations. Therefore, we simply reported whether patients took acetaminophen or NSAIDs (yes/ no) without any minimum dose. However, in some cases the use was less than multiple days per month ( $4 \%$ for acetaminophen and $5 \%$ for NSAIDs, results not shown). In addition, 57 patients $(33 \%)$ suffered from hypertension and $12(7 \%)$ from cardiovascular diseases, both of which are contraindications to NSAIDs use. ${ }^{6}$ This may have resulted in underestimating the use of NSAIDs or Tramadol, as these patients should be excluded from these estimates.

To our knowledge this is the first study that evaluated the full range of combinations of non-surgical treatments for OA, both from the perspective of orthopaedic surgeons and patients. While most recommended nonsurgical treatments for OA were used frequently as single therapy, the combination is used in only a small percentage of OA patients who receive(d) a THA or TKA. Despite their potential for reducing symptoms of knee and hip OA, dietary therapy and physical therapy appear to be least frequently used. By increasing the use of these two non-surgical treatments, primary care physicians and orthopaedic surgeons may be able to help patients better manage their symptoms, thereby improving quality of care and potentially postpone the need for joint arthroplasty, resulting in improved long-term patient outcomes. Future studies should focus on evaluating the reasons (barriers) why some orthopaedic surgeons do not use recommended non-surgical treatments. Such findings may be helpful in developing targeted strategies to improve the use of these treatments in orthopaedic practice and thereby to improve quality of care. Although the recommended non-surgical treatment options have been proven to be effective individually or in combination (eg, physical therapy with dietary therapy $\left.{ }^{24}\right)$, there are no published studies that investigated the combined effect of all of these treatments. Nevertheless, it has been hypothesised that optimised non-surgical treatment could result in significantly 
greater pain reduction, functional improvement and increase in quality of life than usual care in knee OA. ${ }^{32}$ The results from the present study suggest that such better outcomes may be achieved in a considerable part of OA patients.

Acknowledgements The authors are thankful for all the respondents. The authors gratefully acknowledge the input of the Netherlands Orthopaedic Association/Nederlandse Orthopaedische Vereniging (NOV) and the patient associations.

Contributors LVB-V, CHMvdE and TPMVV designed the study. SNH wrote the article and carried out the study. LVB-V and TPMVV supervised the study. LvB-V and PJM-vdM supervised writing of the manuscript. All authors have critically read and modified both the study protocol and previous drafts of the manuscript, and have approved the final version. All authors read and approved the final manuscript.

Funding This work was supported by the Netherlands Organisation for Health Research and Development grant number 837004002 . Funding was obtained from the Netherlands Organisation for Scientific Research (NWO) Incentive fund Open Access publications.

Competing interests None declared.

Ethics approval The Medical Ethical Committee of the Leiden University Medical Center.

Provenance and peer review Not commissioned; externally peer reviewed.

Data sharing statement The relevant anonymised data are available on reasonable request from the authors.

Open Access This is an Open Access article distributed in accordance with the Creative Commons Attribution Non Commercial (CC BY-NC 4.0) license, which permits others to distribute, remix, adapt, build upon this work noncommercially, and license their derivative works on different terms, provided the original work is properly cited and the use is non-commercial. See: http:// creativecommons.org/licenses/by-nc/4.0/

\section{REFERENCES}

1. Bennell KL, Hunter DJ, Hinman RS. Management of osteoarthritis of the knee. BMJ 2012;345:e4934.

2. Zhang W, Nuki G, Moskowitz RW, et al. OARSI recommendations for the management of hip and knee osteoarthritis: part III: changes in evidence following systematic cumulative update of research published through January 2009. Osteoarthritis Cartilage 2010;18:476-99.

3. Hochberg MC, Altman RD, April KT, et al. American College of Rheumatology 2012 recommendations for the use of nonpharmacologic and pharmacologic therapies in osteoarthritis of the hand, hip, and knee. Arthritis Care Res (Hoboken) 2012;64:465-74.

4. Conaghan PG, Dickson J, Grant RL. Care and management of osteoarthritis in adults: summary of NICE guidance. BMJ 2008;336:502-3.

5. Fernandes L, Hagen KB, Bijlsma JW, et al. EULAR recommendations for the non-pharmacological core management of hip and knee osteoarthritis. Ann Rheum Dis 2013;72:1125-35.

6. Netherlands Orthopaedic Association (NOV). Guideline diagnostics and treatment of hip- and knee osteoarthritis. Utrecht: Quality Institute for Health Care (CBO), 2007.

7. Snijders GF, den Broeder AA, van Riel PL, et al. Evidence-based tailored conservative treatment of knee and hip osteoarthritis: between knowing and doing. Scand J Rheumatol 2011;40: 225-31.

8. Shrier I, Feldman DE, Gaudet MC, et al. Conservative non-pharmacological treatment options are not frequently used in the management of hip osteoarthritis. J Sci Med Sport 2006;9 81-6.

9. DeHaan MN, Guzman J, Bayley MT, et al. Knee osteoarthritis clinical practice guidelines-how are we doing? J Rheumatol 2007;34:2099-105.

10. Hunter DJ. Quality of osteoarthritis care for community-dwelling older adults. Clin Geriatr Med 2010;26:401-17.
11. McGlynn EA, Asch SM, Adams J, et al. The quality of health care delivered to adults in the United States. $N$ Engl J Med 2003;348:2635-45.

12. Jordan $\mathrm{KM}$, Sawyer S, Coakley $\mathrm{P}$, et al. The use of conventional and complementary treatments for knee osteoarthritis in the community. Rheumatology (Oxford) 2004;43:381-4.

13. Denoeud L, Mazieres B, Payen-Champenois C, et al. First line treatment of knee osteoarthritis in outpatients in France: adherence to the EULAR 2000 recommendations and factors influencing adherence. Ann Rheum Dis 2005;64:70-4.

14. Nelson AE, Allen KD, Golightly YM, et al. A systematic review of recommendations and guidelines for the management of osteoarthritis: the Chronic Osteoarthritis Management Initiative of the U.S. Bone and Joint Initiative. Semin Arthritis Rheum 2014;43:701-12.

15. Hoogeboom TJ, Snijders GF, Cats HA, et al. Prevalence and predictors of health care use in patients with early hip or knee osteoarthritis: two-year follow-up data from the CHECK cohort. Osteoarthritis Cartilage 2012;20:525-31.

16. Smink AJ, Dekker J, Vliet Vlieland TP, et al. Health care use of patients with osteoarthritis of the hip or knee after implementation of a stepped care strategy: an observational study. Arthritis Care Res (Hoboken) 2014;66:817-27.

17. Li LC, Maetzel A, Pencharz JN, et al. Use of mainstream nonpharmacologic treatment by patients with arthritis. Arthritis Rheum 2004;51:203-9.

18. Brand CA, Harrison C, Tropea J, et al. Management of osteoarthritis in general practice in Australia. Arthritis Care Res (Hoboken) 2013;66:551-8.

19. Nilsdotter AK, Toksvig-Larsen S, Roos EM. Knee arthroplasty: are patients' expectations fulfilled? A prospective study of pain and function in 102 patients with 5-year follow-up. Acta Orthop 2009;80:55-61.

20. Anderson JG, Wixson RL, Tsai D, et al. Functional outcome and patient satisfaction in total knee patients over the age of 75 $J$ Arthroplasty 1996;11:831-40.

21. Baker PN, van der Meulen JH, Lewsey J, et al. The role of pain and function in determining patient satisfaction after total knee replacement. Data from the National Joint Registry for England and Wales. J Bone Joint Surg Br 2007;89:893-900.

22. Gandhi R, Davey JR, Mahomed NN. Predicting patient dissatisfaction following joint replacement surgery. $J$ Rheumatol 2008:35:2415-18

23. McHugh GA, Luker KA, Campbell M, et al. A longitudinal study exploring pain control, treatment and service provision for individuals with end-stage lower limb osteoarthritis. Rheumatology (Oxford) 2007;46:631-7.

24. Messier SP, Loeser RF, Miller GD, et al. Exercise and dietary weight loss in overweight and obese older adults with knee osteoarthritis: the Arthritis, Diet, and Activity Promotion Trial. Arthritis Rheum 2004:50:1501-10

25. Dutch Arthroplasty Register (LROI). More insights into quality of orthopaedic care (in Dutch). 's Hertogenbosch, Netherlands Orthopaedic Association: 2012

26. van den Ende CM, Bierma-Zeinstra SM, Vlieland TP, et al. Conservative treatment of hip and knee osteoarthritis: a step-by-step treatment strategy. Ned Tijdschr Geneeskd 2010;154:A1574.

27. Smink AJ, van den Ende $\mathrm{CH}$, Vliet Vlieland TP, et al. "Beating osteoARThritis": development of a stepped care strategy to optimize utilization and timing of non-surgical treatment modalities for patients with hip or knee osteoarthritis. Clin Rheumatol 2011;30:1623-9.

28. Keurenties JC, Fiocco M, So-Osman C, et al. Hip and knee replacement patients prefer pen-and-paper questionnaires: Implications for future patient-reported outcome measure studies. Bone Joint Res 2013;2:238-44.

29. Ursum J, Hek K, Spronk I, et al. How common is osteoarthritis, and for whom? (in Dutch). NIVEL Zorgregistraties eerste www.nivel.nl/ node/3069 (accessed August 2015)

30. Voorn VM, Marang-van de Mheen PJ, Wentink MM, et al, LISBOA Study Group. Perceived barriers among physicians for stopping non-cost-effective blood-saving measures in total hip and total knee arthroplasties. Transfusion 2014;54(10 Pt 2):2598-607.

31. Hofstede SN, van Bodegom-Vos L, Wentink MM, et al. Most important factors for the implementation of shared decision making in sciatica care: ranking among professionals and patients. PLOS ONE 2014:9:e94176.

32. Skou ST, Roos EM, Laursen MB, et al. Efficacy of multimodal, systematic non-surgical treatment of knee osteoarthritis for patients not eligible for a total knee replacement: a study protocol of a randomised controlled trial. BMJ Open 2012;2: doi:10.1136/ bmjopen-2012-002168 